\title{
Timing-Jitter Tolerant Nyquist Pulse for Terahertz Communications
}

This paper was downloaded from TechRxiv (https://www.techrxiv.org).

\section{LICENSE}

CC BY-NC-SA 4.0

SUBMISSION DATE / POSTED DATE

02-08-2021 / 03-08-2021

\section{CITATION}

Shehata, Mohamed; Wang, Ke (Desmond); Webber, Julian; Fujita, Masayuki; Nagatsuma, Tadao; Withayachumnankul, Withawat (2021): Timing-Jitter Tolerant Nyquist Pulse for Terahertz Communications. TechRxiv. Preprint. https://doi.org/10.36227/techrxiv.15088575.v1

$\mathrm{DOI}$ 


\title{
Timing-Jitter Tolerant Nyquist Pulse for Terahertz Communications
}

\author{
Mohamed Shehata, Senior Member, IEEE, Ke Wang, Senior Member, IEEE, Julian Webber, Senior \\ Member, IEEE, Masayuki Fujita, Member, IEEE, Tadao Nagatsuma, Fellow, IEEE, \\ and Withawat Withayachumnankul, Senior Member, IEEE
}

\begin{abstract}
Seamless integration of terahertz wireless communications with existing optical access networks can potentially offer tens of gigahertz of channel bandwidth. However, the timingjitter exhibited by the electronics-based transceivers is a key transmission impairments that tends to ceil the performance of these hybrid access networks, especially at high transmission baud rates. In this work, we propose a sinc-Lorentzian Nyquist pulse shape that outperforms the conventional raised-cosine and the better-than raised-cosine pulse shapes under the influence of timing-jitter up to $35 \%$ of the symbol duration. Experimental demonstrations are carried out using a $311 \mathrm{GHz}$ photonicterahertz system operating at a standard bit rate of $1.44 \mathrm{Gbit} / \mathrm{s}$ to investigate the robustness of the three pulse shapes against timing-jitter. It is confirmed that the proposed pulse shape is highly-tolerant to timing-jitter as well as the nonlinearity exhibited by terahertz systems, and hence, can improve the bit error rate performance by an order of magnitude when the average timing-jitter is as large as $0.24 \mathrm{~ns}$. The proposed pulse shape is a critical step for designing ultra-high-speed terahertz communications links with improved robustness to timing-jitter.
\end{abstract}

Index Terms-Optical pulse shaping, radio-over-fiber, terahertz communications, terahertz over fiber, terahertz photonics, timing-jitter, waveform design.

\section{INTRODUCTION}

D AY-by-day increasing demands for reliable high-speed wireless services are expected to rapidly approach and exceed the capacity of fifth-generation $(5 \mathrm{G})$ communications networks. These demands are unlikely to be fulfilled without ongoing vigorous research on next-generation wireless networks, namely, 6G. A key enabler for $6 \mathrm{G}$ communications is terahertz technology that offers ample spectral resources for point-to-point wireless systems [1]. Numerous advantages

Mohamed Shehata and Withawat Withayachumnankul are with the Terahertz Engineering Laboratory, School of Electrical and Electronics Engineering, The University of Adelaide, Adelaide, 5005 SA e-mail: (mohamed.shehata@adelaide.edu.au; withawat@adelaide.edu.au).

Ke Wang is with the School of Engineering, RMIT University, Melbourne, Victoria, Australia. e-mail: (ke.wang@rmit.edu.au).

Julian Webber, Masayuki Fujita, and Tadao Nagatsuma are with the Graduate School of Engineering Science, Osaka University, Osaka, Japan. e-mail: (nagatuma@ee.es.osaka-u.ac.jp; fujita@ee.es.osaka-u.ac.jp; webber@ee.es.osaka-u.ac.jp).

Manuscript received Month XX, 2021; revised Month XX, 2021; accepted Month XX, 2021. Date of publication Month XX, 2021; date of current version Month XX, 2021. The authors wish to acknowledge support from the Australian Research Council Discovery Project (No. ARC DP180103561), The Australian Government through the Australian Government Research Training Program Scholarship, The University of Adelaide through the Adelaide Scholarship International, the SmartSAT Cooperative Research Centre (SmartSat CRC) through the SmartSat Ph.D. top-up scholarship (Project No.: P1-14s), and the Core Research for Evolutional Science \& Technology (CREST) program of the Japan Science and Technology Agency (JST) (JPMJCR1534). are expected from pushing the carrier frequencies towards the terahertz band (100 GHz to $10 \mathrm{THz})$, such as increased channel capacities with much lower latency and increased user activities on the network. In practice, the generation and modulation of such high carrier frequencies is usually accompanied by a randomly fluctuating phase noise, which becomes noticeable as random timing-jitter in the baseband modulated signal. Unless precisely estimated and compensated, this timing-jitter gives rise to imperfect synchronization, i.e., a net timing-offset, between the transmitter and the receiver, which could severely deteriorate the end-to-end bit error rate (BER) performance, especially when increasing the system data rate [2].

In the field of terahertz communications, timing-jitter with a remarkable proportion of the symbol period has been frequently observed in many experimental demonstrations [3][6]. For instance, in a typical terahertz electronic receiver, the average timing-jitter was measured to be in the range of $10 \mathrm{ps}$ at $5 \mathrm{Gbit} / \mathrm{s}$ to $25 \mathrm{ps}$ at $12 \mathrm{Gbit} / \mathrm{s}$ [6], which corresponds to about $5 \%$ to $30 \%$ of the symbol duration. The impact of this timingjitter, and consequently, the inter-symbol interference (ISI), on the BER performance was considered insignificant since the received signal exhibited a highly-confined Gaussian-like profile over the allocated symbol duration [6]. However, for the same symbol rate, the root mean squared (rms) bandwidth of a typical Gaussian pulse is much higher than the Nyquist bandwidth requirement. Therefore, in most of the previous demonstrations of terahertz communications, e.g., [7]-[10], it has been a common practice to rely on the raised-cosine (RC) pulse shape with various roll-off factors for bandwidthefficient signaling.

Although spectrally-efficient, the transmission performance of Nyquist pulses, including the RC pulse, is sensitive to the timing-jitter due to the large overlap between adjacent pulses. The ISI caused by this overlap can severely deteriorate the BER performance unless the detection is performed at the optimum sampling instants at the receiver side. In [11], it has been shown that when the timing-offset is $25 \%$ of the symbol period, the BER can exceed $10^{-3}$, which is much higher than the error-free transmission limit of $10^{-12}$ [12].

Admittedly, in most of the previous demonstrations of terahertz communications links, the impact of timing-jitter on the transmission performance of RC pulses has not been considered since offline digital signal processing (DSP) techniques have been employed to compensate for various transmission impairments, including the timing-jitter. Whilst offline DSP techniques are sufficient for proof-of-concept experiments, 
the timing-jitter estimation and compensation can be a complicated DSP task for receivers with limited computational resources. This issue can be challenging in practical scenarios, especially for real-time applications.

A spectrally-efficient solution to reduce the severity of the timing-jitter on the transmission performance of Nyquist pulses is to design rapidly decaying pulses in order to reduce the power contained in time-domain sidelobes and, consequently, the resulting ISI. This way, the BER of these pulses can be improved and the requirement for high-speed precise timing-control circuitry at the receiver side can be relaxed.

In this work, we propose a sinc-Lorentzian Nyquist pulse shape that is highly-tolerant to timing-jitter exhibited by photonic-terahertz systems. The proposed pulse shape possesses two independent degrees of freedom, which allow for flexible control of its temporal and spectral characteristics. We further investigate the impact of timing-offsets on the transmission and detection performance of the proposed waveform in comparison to the other two common waveforms using a photo-mixing-based terahertz communications link. It should be highlighted that a relevant non-Nyquist waveform was presented in [13] without considering the Nyquist condition for ISI-free signaling.

The rest of this paper is organized as follows. Section II overviews the temporal and spectral characteristics of the RC and BTRC waveforms as potential candidates for terahertz communications. The proposed Nyquist pulse is presented in Section III. Here, an ISI analysis is carried out for the three considered pulse shapes in Section IV. Section V presents an experimental investigation of the transmission performance of these pulse shapes.

\section{Conventional WAVEForms For Terahertz COMMUNICATIONS}

Nyquist pulse shapes, including the RC pulse, are inherently descendants of the sinc pulse with different decaying envelope profiles. The sinc pulse has zero-crossings at integer multiples of the symbol duration [14]. This property satisfies the first Nyquist criterion to minimize the impact of ISI on the BER performance in the presence of transmitter-receiver synchronization errors [15]. The modified envelopes, on the other hand, are designed to improve the temporal and spectral characteristics of the mother sinc pulse, namely, the decay rate in the time-domain and the stop-band attenuation in the frequency-domain. Various Nyquist pulses with different envelope functions have been proven to outperform the RC pulse in band-limited electrical channels, such as the generalized phase-compensated Nyquist pulse [14], the better-than raised-cosine (BTRC) pulse [16], the flipped hyperbolic-secant (fsech) and the flipped inverse hyperbolic-secant pulses [17]. Of particular importance is the BTRC pulse as it is the only pulse, other than the RC pulse, that has a closed-form timedomain expression [16], which facilitates the theoretical evaluation of the ISI. Moreover, the BTRC pulse has been proven to improve the BER performance in generalized frequency division multiplexing systems and visible light communications systems [18], [19]. Therefore, the RC and BTRC pulses are chosen here as comparison benchmarks.
The spectrum of a typical RC waveform is given by [16]

$$
\begin{aligned}
& P_{\mathrm{RC}}(f)= \\
& \begin{cases}1, & 0 \leq|f|<B \alpha^{-} \\
\frac{1}{2}\left\{1+\cos \left(\frac{\pi}{2 B \alpha}\left(|f|-B \alpha^{-}\right)\right)\right\}, & B \alpha^{-} \leq|f| \leq B \alpha^{+} \\
0, & B \alpha^{+} \leq|f|,\end{cases}
\end{aligned}
$$

where $\alpha^{+} \triangleq(1+\alpha), \alpha^{-} \triangleq(1-\alpha), \alpha$ is the roll-off factor, $T_{s}$ is the symbol period, and $B=1 /\left(2 T_{s}\right)$ is the Nyquist bandwidth. The corresponding RC time-domain waveform is given by [16].

$$
p_{\mathrm{RC}}(t)=\operatorname{sinc}\left(\frac{t}{T_{s}}\right) \frac{\cos \left(2 \pi \alpha t / T_{s}\right)}{1-4 \alpha^{2} t^{2} / T_{s}^{2}} .
$$

The spectrum of the BTRC pulse is given by [16]:

$$
\begin{aligned}
& P_{\text {BTRC }}(f)= \\
& \begin{cases}1, & 0 \leq|f|<B \alpha^{-} \\
\exp \left\{\frac{\ln 2}{\alpha B}\left|f-B \alpha^{-}\right|\right\}, & B \alpha^{-} \leq|f|<B \\
1-\exp \left\{\frac{\ln 2}{\alpha B}\left|f-B \alpha^{+}\right|\right\}, & B \leq|f|<B \alpha^{+} \\
0, & B \alpha^{+} \leq|f| .\end{cases}
\end{aligned}
$$

The corresponding time-domain pulse is given by [16]: $p_{\mathrm{BTRC}}(t)=$

$2 B \operatorname{sinc}(2 B t) \times \frac{4 \beta \pi t \sin (2 \pi B \alpha t)+2 \beta^{2} \cos (2 \pi B \alpha t)-\beta^{2}}{4 \pi^{2} t^{2}+\beta^{2}}$,

where $\beta=(\ln 2 / \alpha B)$. It should be pointed out that, practically, the RC and the BTRC pulse shaping is realized using both the transmitter and the receiver sides [20]. Each side employs the square root of (1) and (3).

\section{Proposed Nyquist Pulse Shape}

In this work, the proposed pulse shape, referred as the sincLorentzian pulse, is defined mathematically as follows:

$$
p_{\mathrm{SL}}(t)=\pi \exp \left(\gamma B \alpha^{-}\right) \exp \left(-\gamma \frac{\alpha^{-}}{T_{s}}\right) \operatorname{sinc}\left(\frac{t}{T_{s}}\right) E(t)
$$

where $\gamma$ is a parameter that controls the time-domain decay rate as well as the spectral distribution of $p_{\mathrm{SL}}(t)$, and $E(t)$ is the envelope of $p_{\mathrm{SL}}(t)$, which is defined as follows:

$$
E(t)=\frac{\alpha^{-} \operatorname{sinc}\left(\frac{\alpha^{-} t}{T_{s}}\right)}{T_{s}}+\frac{\cos \left(\frac{\pi \alpha^{-} t}{T_{s}}\right)-\left(\frac{\pi t}{\gamma}\right) \sin \left(\frac{\pi \alpha^{-} t}{T_{s}}\right)}{\gamma+\left(\frac{\pi^{2} t^{2}}{\gamma}\right)} .
$$

The two-parameters in (5), i.e., $\alpha^{-}$and $\gamma$, make it possible to flexibility control the temporal and the spectral characteristics of the sinc-Lorentzian pulse [21]. 


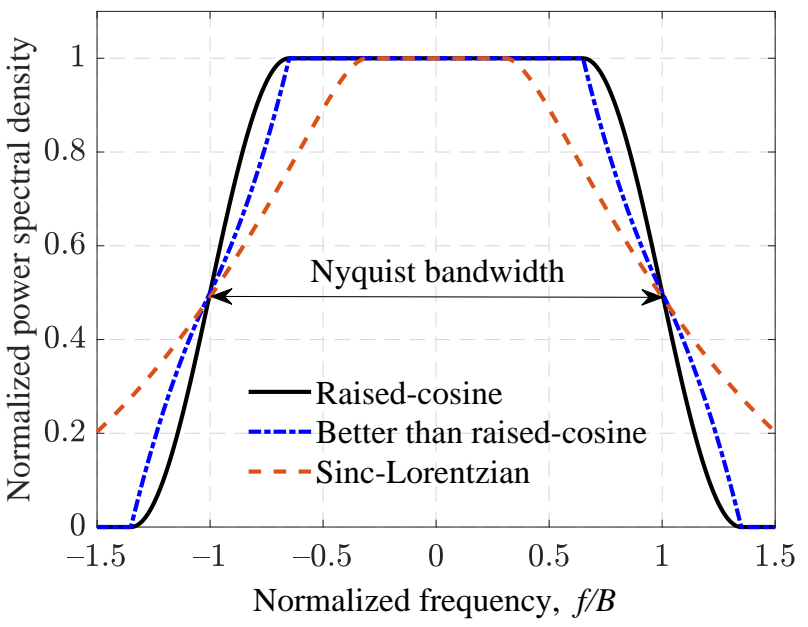

Fig. 1. Spectra of the three considered waveforms, evaluated at a roll-off factor of $\alpha=0.35$. For the sinc-Lorentzian pulse, $\gamma=7.226 \times 10^{-9}$.

The spectrum of (5) is numerically evaluated using the fast Fourier transform (FFT) technique with $2^{18}$ points. Figure 1 shows the spectra of the proposed waveform. The RC and BTRC spectra are also shown for comparison. The spectra of the three waveforms are evaluated at the same roll-off factor of $\alpha=0.35$. For the sinc-Lorentzian pulse, $\gamma=7.226 \times 10^{-9}$.

Figure 2 shows the eye diagrams of the three considered waveforms evaluated using $2^{10}$ BPSK symbols at $\alpha=0.35$. The proposed waveform has much smaller amplitude distortions, i.e., lower sidelobe energy, and a much wider horizontal eye opening at the receiver side than the eye diagrams of the classical RC and BTRC Nyquist pulses. This implies that the proposed pulse can tolerate a larger timing deviation around the optimum sampling instant without experiencing significant ISI. This behavior is attributed to the pulse decay rate in the time domain, which we discuss in details below.

In [17], it has been shown that the decay rate of a Nyquist waveform depends on the overall waveform design, i.e., considering the mother sinc together with its envelope, rather than the particular envelope decay order. For instance, analytically, (2) and (4) show that the envelopes of the RC and the BTRC pulses decay as $|t|^{-3}$ and $t^{-2}$, respectively. Also, the two pulse shapes presented in [17] show an analytical envelope decay rate of $|t|^{-3}$ and $t^{-2}$. However, the overall waveform amplitude of the main sidelobes with the $t^{-2}$ envelope decay rate are smaller than those of the pulse shapes with the $|t|^{-3}$ envelope decay rate. Likewise, although Eq. 5. 5hows that the proposed pulse shape has an envelope asymptotic decay of $t^{-2}$, Figs. 3 . a) and 3 (b) show that this decay rate is larger than the RC and the BTRC pulses. Further to that, since we employ a photonics-based terahertz communications system in this work, the impact of the EO modulation nonlinearity on the three considered waveforms is illustrated in Fig. 3.c). This figure plots the optical waveforms corresponding to the electrical waveform in Fig. 3(a) for $0 \leq t \leq 3 T_{s}$. Since the optical intensity is proportional to the squared waveform amplitude, the three optical waveforms decay more rapidly compared to the corresponding electrical waveforms in Fig.
3 a). In particular, the decay rate is doubled due to the EO modulation. Additionally, the inset of Fig. 3(c) shows that the maximum sidelobe level of the proposed waveform, i.e., for $T_{s} \leq t \leq 2 T_{s}$, is much lower than the RC and the BTRC waveforms. To put into perspective, the maximum sidelobe optical intensity of the proposed pulse is lower than the corresponding levels of the RC and the BTRC pulses by $17 \mathrm{dBr}$ and $15 \mathrm{dBr}$, respectively.

\section{INTER-SYMBOL INTERFERENCE ANALYSIS}

Assuming ideal sampling and binary phase shift keying (BPSK) modulation, the received symbol sequence is ISIfree and the probability of bit error is theoretically given by $P_{\varepsilon}=Q(\sqrt{2 \mathrm{SNR}})$, where $\mathrm{SNR}$ is the signal-to-noise ratio (SNR), and $Q($.$) is the complementary cumulative distribu-$ tion function of a standard zero-mean unit-variance Gaussian distribution. At an SNR of $15 \mathrm{~dB}, P_{\varepsilon}=9.124 \times 10^{-16}$, which is much lower than the standard error-free transmission limit of $10^{-12}[12]$. In the presence of timing-jitter, the semianalytical method proposed in [11] can be used to evaluate the ISI performance of an arbitrary Nyquist pulse. Here, to avoid the impact of the residual errors associated with the approach in [11], we employ a more straightforward semi-analytical approach to quantify the impact of the timing-jitter on the probability of symbol error for the considered pulse shapes. First, we formulate the received symbol sequence, denoted by $y(t)$, in the presence of timing-jitter and the additive white Gaussian noise (AWGN), as follows:

$$
y(t)=\sum_{k=-N^{-}}^{N^{+}} b_{k} p\left(t \pm \Delta t_{k}-k T_{s}\right)+n(t),
$$

where $k \in\{0,1,2,3 \ldots\}$ is the symbol index, $N^{-}$is the number of interfering symbols that precedes the central data symbol located at $t=0$, and $N^{+}$denotes the number of symbols that follow this central symbol. Moreover, $b_{k} \in\{ \pm 1\}$ is the $k^{\text {th }}$ BPSK information symbol, and $n(t)$ is the AWGN. The pulse shape $p(t)$ denotes one of the three candidate pulses considered here: $p_{\mathrm{RC}}(t), p_{\mathrm{BTRC}}(t)$ or $p_{\mathrm{SL}}(t)$ and $\Delta t_{k}$ denotes the timing-jitter introduced to the $k^{\text {th }}$ symbol. A \pm sign is introduced to $\Delta t_{k}$ in (7) in order to account for positive and negative timing deviations around the optimum sampling instant of the $k^{\text {th }}$ symbol. Secondly, we assume that $y(t)$ is forwarded to a timing-jitter estimator and compensator. The compensated received symbol is denoted by $\hat{y}(t)=y\left(t \mp \widehat{\Delta t_{k}}\right)$, where $\widehat{\Delta t_{k}}$ is the estimated timing-jitter. At the optimum sampling instants, i.e., $t=k T_{s}, \hat{y}(t)$ is given by

$$
\hat{y}\left(k T_{s}\right)=p\left( \pm \delta t_{k}\right) b_{k}+I_{k}\left(\delta t_{m}\right)+n_{k},
$$

where $p\left( \pm \delta t_{k}\right)$ accounts for the reduction in the desired symbol amplitude, and $I_{k}\left(\delta t_{m}\right)$ is the adjacent ISI, defined as follows:

$$
I_{k}\left(\delta t_{m}\right) \triangleq \sum_{m=-N^{-}}^{N^{+}} b_{m} p\left( \pm \delta t_{m}+(k-m) T_{s}\right) ; m \neq k .
$$

Morevoer, $\delta t_{k}=\left|\widehat{\Delta t_{k}}-\Delta t_{k}\right|$ is the timing-jitter estimation error, while $n_{k}$ is the AWGN at the $k^{\text {th }}$ symbol. A decision variable, denoted by $z_{k}$, is formed as follows: 

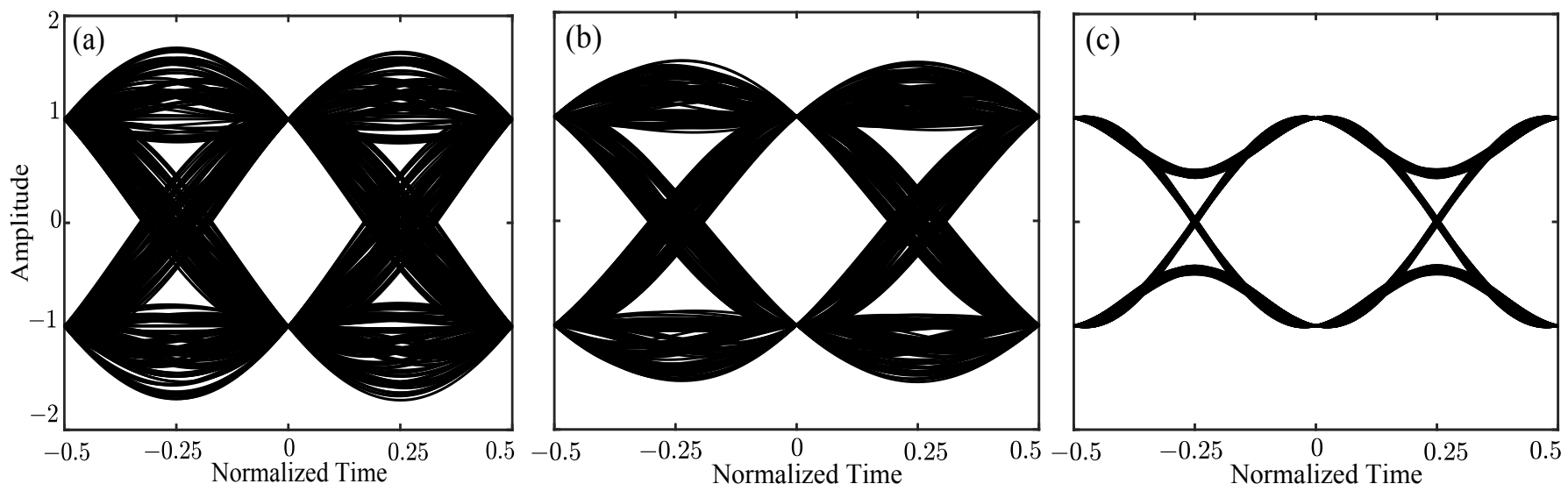

Fig. 2. Eye diagrams of a binary-phase modulated sequence of $2^{10}$ symbols length. (a) Raised-cosine pulse with $\alpha=0.35$, (b) better-than raised-cosine pulse with $\alpha=0.35$, and (c) proposed sinc-Lorentzian pulse shape with $\alpha=0.35$ and $\gamma=7.226 \times 10^{-9}$.
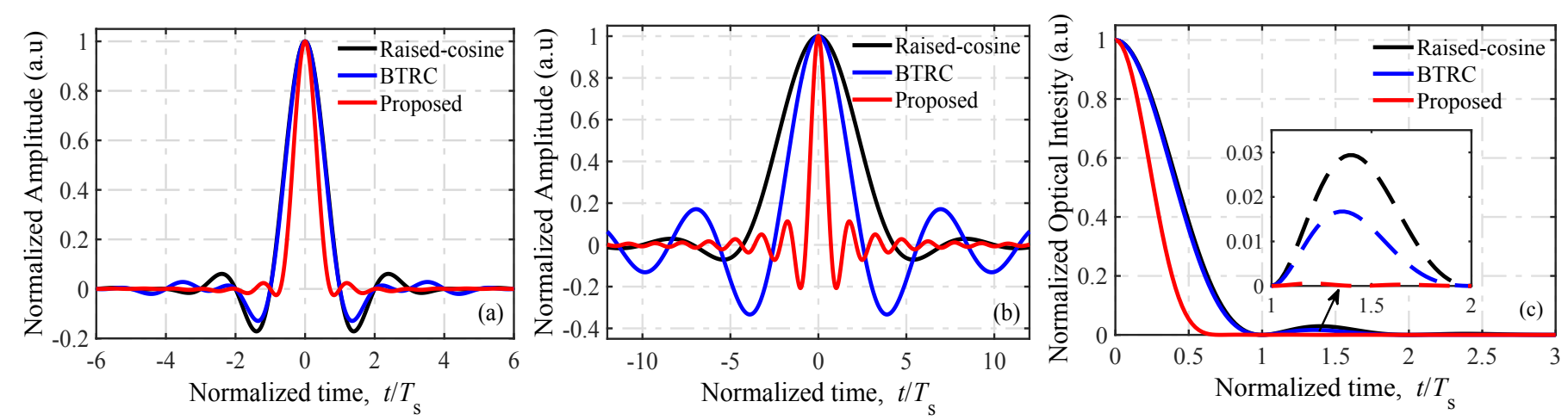

Fig. 3. Simulated waveforms. (a) Electrical baseband waveforms, (b) envelopes of the electrical baseband waveforms, and (c) optical intensity waveforms. The roll-off factor is fixed at $\alpha=0.35$ for the raised-cosine and the BTRC waveforms, whereas, for the proposed waveform, $\alpha=0$ and $\gamma=7.226 \times 10^{-9}$.

$$
z_{k}= \begin{cases}+1 ; & \hat{y}\left(k T_{s}\right)>0 \\ -1 ; & \hat{y}\left(k T_{s}\right)<0\end{cases}
$$

It should be highlighted that, $p\left( \pm \delta t_{k}\right)=1$ and $I_{k}\left( \pm \delta t_{m}\right)=$ 0 when $\delta t_{k}=0$, whereas for $\delta t_{k} \neq 0$, the values of $p\left( \pm \delta t_{k}\right)$ and $I_{k}\left( \pm \delta t_{m}\right)$ depend on the particular choice of the pulse shape, $p(t)$. Thirdly, the probability of symbol error, denoted by $P_{\varepsilon}$, is numerically evaluated by calculating $\operatorname{Pr}\left\{z_{k} \neq b_{k}\right\}$ using the Monte-Carlo numerical simulation technique. It is noteworthy that, the proposed approach results in the same error performance as the earlier approach [11] when used to evaluate time unlimited waveforms [16], [17], while it can avoid the mathematical complexity associated with the approach in [11].

Table I lists the simulation parameters used to evaluate the BER performance of the three considered waveforms in the presence of timing-jitter. The symbol rate, $R_{s}$, corresponds to a standard symbol rate of $1.44 \mathrm{Gbit} / \mathrm{s}$ [12]. Additionally, in practice, $p(t)$ is the impulse response of a digital FIR filter, which is implemented using a finite number of symmetric taps to avoid long processing delays [22]. Therefore, the impact of ISI introduced by a finite number of interfering symbols before and after the desired symbol is considered in (7). The number of interfering symbols listed in Table $\mathrm{I}$ is in line with the typical filter half-length required for a $(2 L+1)$-tap symmetric FIR filter [22], where $L \leq 31$.

Figure (4) plots the simulated BER performance of the three considered waveforms in the presence of timing-offsets. For a given pulse shape with a fixed roll-off factor, the BER deteriorates with an increase in the timing-offset. Moreover, for the RC and BTRC pulse shapes and fixed timing-offset, increasing the roll-off factor improves the BER performance. This is because increasing the roll-off factor increases the bandwidth beyond the Nyquist bandwidth and hence, reduces the time-domain sidelobes, which in turn reduces the ISI. On the other hand, for the proposed sinc-Lorentzian pulse, increasing the roll-off factor degrades the BER performance. This is because the sinc pulse envelope in (5) depends on $\alpha^{-}$ rather than $\alpha$. Nevertheless, among the three pulse shapes,

TABLE I

SiMUlation PARAMETERS.

\begin{tabular}{ccc}
\hline Parameter & Value & Unit \\
\hline Roll-off factor, $\alpha$ & $0.25,0.35,0.45$ & - \\
Spectral decay factor, $\gamma$ & $7.226 \times 10^{-9}$ & $\mathrm{~s}^{-1}$ \\
Symbol rate, $R_{s}$ & 1.44 & $\mathrm{Gbit} / \mathrm{s}$ \\
Symbol period, $T_{s}$ & 0.694 & $\mathrm{~ns}$ \\
Normalized timing-offset, $\delta t_{k} / T_{s}$ & $\pm\{0.2,0.25,0.3,0.35\}$ & - \\
Number of ISI symbols, $N^{+}, N^{-}$ & 40 & - \\
\hline
\end{tabular}



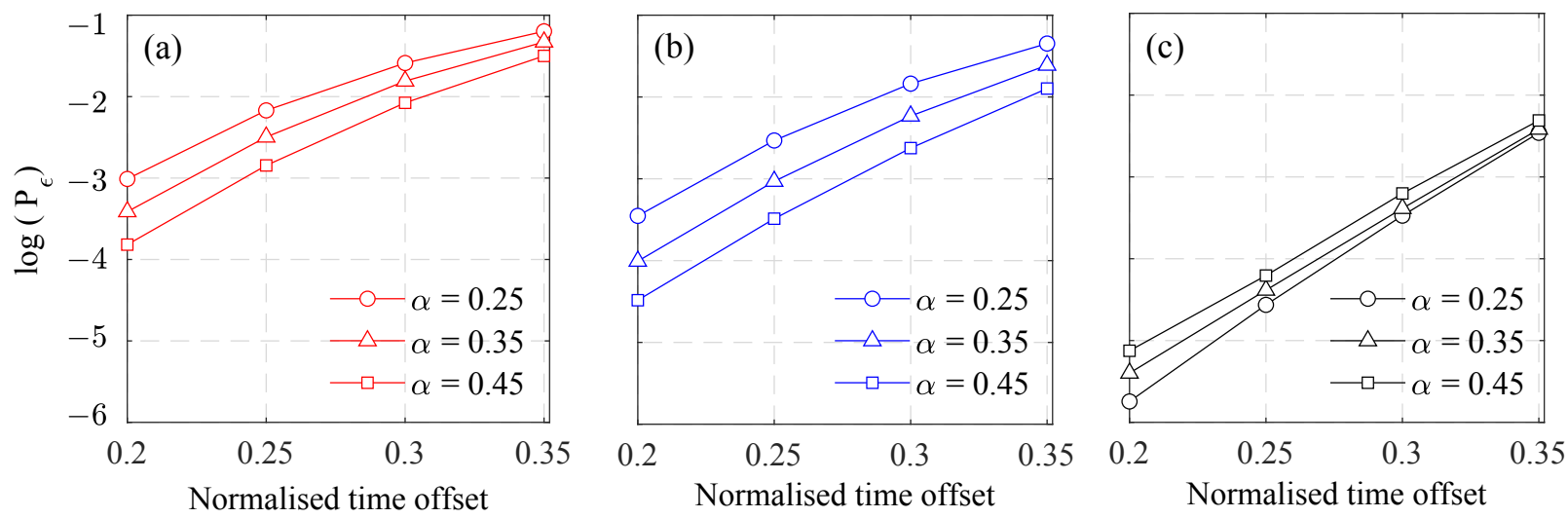

Fig. 4. Probability of bit error performance resulting from the timing-offset-induced inter-symbol interference for (a) Raised-cosine pulse, (b) better-than raised-cosine pulse, and (c) proposed sinc-Lorentzian pulse shape. The roll-off factor, $\alpha$, is $0.25,0.35$, and 0.45 . For the proposed waveform, $\gamma=7.226 \times 10^{-9}$.

the BER performance of the sinc-Lorentzian pulse is least sensitive to the roll-off factor as well as the most tolerant to the timing-offsets. The differences in the BER performances exhibited by the three pulse shapes can be explained in terms of the effective SNR of the timing-offset-compensated signal in (8). Assuming a Gaussian distribution for the ISI in (8), the effective SNR becomes equal to the signal-to-interference plus noise ratio. In this case, the non-zero error performance of timing-offset estimators, i.e., when $\delta t_{k} \neq 0$, in the presence of AWGN is lower-bounded by the modified Cramer-Rao bound (MCRB) [23] and can be formulated in terms of the effective SNR as follows:

$$
\mathrm{SNR}_{\mathrm{eff}} \geq \frac{1}{4 \pi^{2} \sigma_{f} \sigma_{t}},
$$

where $\mathrm{SNR}_{\text {eff }}$ is the effective SNR of the timing-offsetcompensated signal, while $\sigma_{f}$ and $\sigma_{t}$ are the root mean squared (RMS) spectral width and temporal width, respectively. The derivation of (11) from the MCRB in [23] is detailed in the Appendix of this work. As can be seen from (11), the effective SNR of a signaling waveform, and its corresponding BER performance, depends on the distribution of its energy in the time and the frequency domains. Figure (5) illustrates normalized time-bandwidth product (TBP), defined as $\sigma_{t} \sigma_{f} /\left(B T_{\mathrm{s}}\right)$, for the three considered waveforms when $0 \leq \alpha \leq 1$. Figure (5) also plots the corresponding $\mathrm{SNR}_{\text {eff }}$ over the same range of $\alpha$ according to (11). In both plots, the sinc-Lorentzian pulse is calculated at $\gamma=7.226 \times 10^{-9}$. In terms of the SNR, the three pulse shapes are equivalent when $\alpha$ is about 0.8 . On one hand, decreasing $\alpha$ below 0.8 improves the SNR of the sincLorentzian pulse compared to the RC and the BTRC pulses. This improvement attains its maximum, which is about $12 \mathrm{~dB}$, at $\alpha=0$. On the other hand, increasing $\alpha$ beyond 0.8 reverses the situation as the SNR of the sinc-Lorentzian pulse becomes lower than that of the RC or the BTRC pulse. However, the maximum degradation in the SNR does not exceed $2 \mathrm{~dB}$.

\section{EXPERIMENTAL RESUlts AND ANALYSIS}

To assess the transmission performance of the three considered pulse shapes, we conduct experimental investigations using the same experimental setup, together with the DSP at the transmitter side, as that reported in [13]. Figure 6 shows this experimental setup as well as the post-detection DSP at the receiver side for this work.

At the receiver side, the baseband envelope of the received terahertz signal is detected via the Schottky-barrier diode (SBD) and the free space path loss is compensated by a $30 \mathrm{~dB}$ low-noise amplifier (LNA). The amplified signal is then sampled by a $80 \mathrm{GSample/s}$ real-time oscilloscope (RTO) for offline DSP as illustrated in Fig. 6. To match the sampling rate at the transmitter side, the recorded data is down-sampled from $80 \mathrm{GSample/s}$ to $64 \mathrm{GSample/s} \mathrm{using} \mathrm{a} \mathrm{down-sampling}$ ratio of 5:4 to remove the redundant samples. To mitigate the AWGN, a 44-taps moving average (MA) filter is applied to the received signal as a denoising filter. This filter length is equivalent to the width of the symbol duration, measured in samples, to ensure that the AWGN is averaged over the symbol duration without influencing adjacent symbols. The Tx-Rx synchronization is recovered by estimating the optimum delay

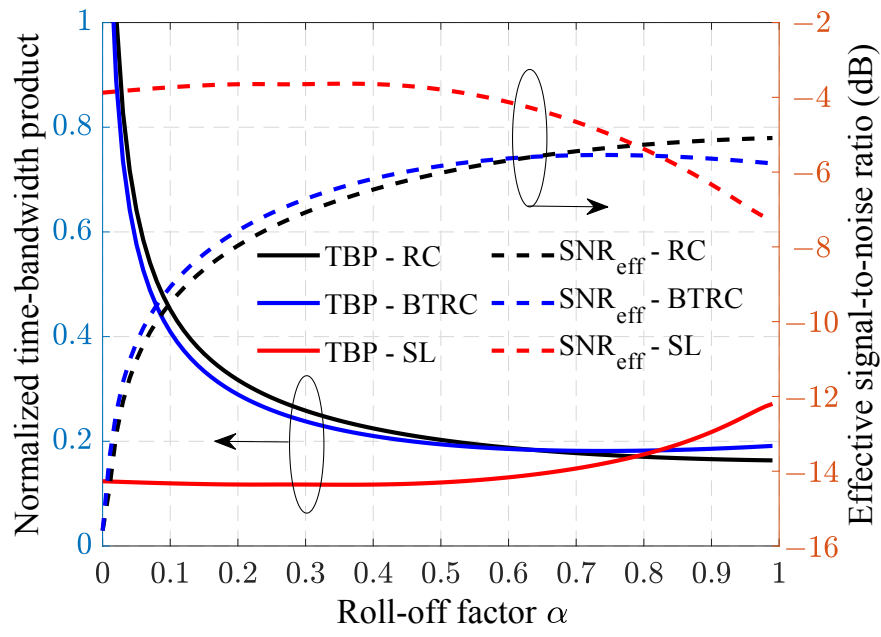

Fig. 5. Time-bandwidth product and effective signal-to-noise ratio versus the roll-off factor, $\alpha$. The time-bandwidth product is normalized to the Nyquist bandwidth multiplied by the symbol duration. TBP: time-bandwidth product. RC: raised-cosine. BTRC: better-than raised-cosine. SL: sinc-Lorentzian. The SL spectrum is evaluated at $\gamma=7.226 \times 10^{-9}$. 


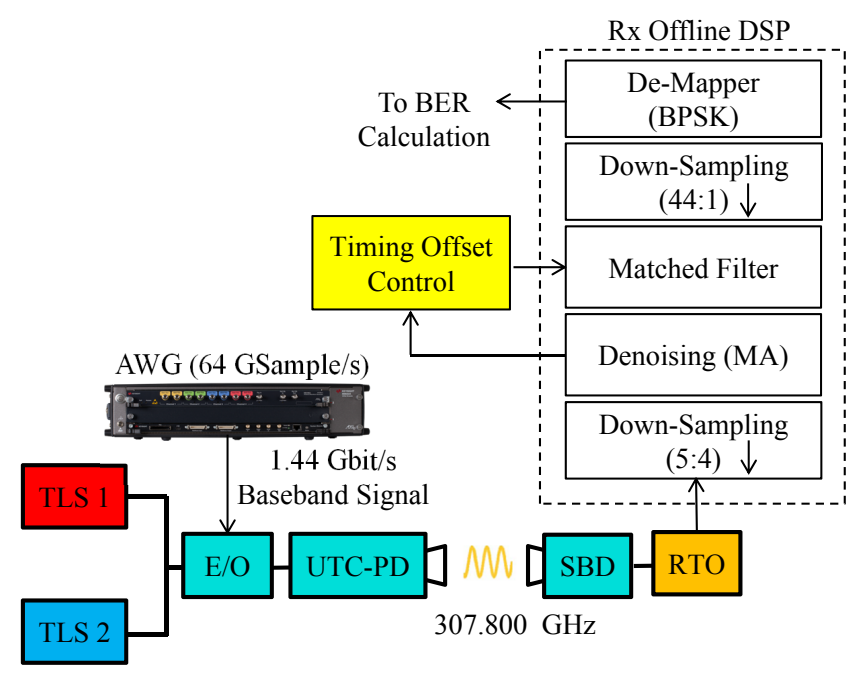

Fig. 6. Block diagram of the experimental setup including the receiver processing. TLS: tunable laser source. AWG: arbitrary waveform generator. E/O: electro-optic. SBD: Schottky-barrier diode. UTC-PD: uni-traveling carrier photo-diode. RTO: real-time oscilloscope. MA: moving average. Rx DSP: receiver digital signal processing. The maximum sampling rate of the AWG is $65 \mathrm{GS} / \mathrm{s}$.

that maximizes the cross-correlation of the transmitted and the received sequences. Afterwards, the timing-offset process is imitated offline by introducing an integer delay of $\left[\delta t / T_{\mathrm{s}}\right]$ samples to the time-corrected signal before matched filtering, where [.] is the rounding operator.

Figures 7 (a) and (b) plot the BER performance of the three considered pulse shapes versus the sampling time-offset, $\delta t$, at a photocurrent of $I_{\mathrm{ph}}=3 \mathrm{~mA}$ or $5 \mathrm{~mA}$, which corresponds to an emitted terahertz power of $P_{\mathrm{THz}}=-18.4 \mathrm{dBm}$ or $-13.3 \mathrm{dBm}$, respectively. The eye diagrams are plotted for the three waveforms at $\delta t=0.05 T_{s}$. As shown in the BER plots, for the three pulse shapes, the BER increases monotonically with increasing the timing-offset. This BER deterioration is a result of the reduction in the desired symbol amplitude and an accompanying increase in the sidelobe level introduced by the adjacent symbols as the sampling instant is displaced further from the optimum sampling instant.

Figure 7(a) shows that, at $\delta t=0.35 T_{s}$, the BER performance of the terahertz link is improved by a factor of $50 \%$ when the RC and BTRC pulses are replaced by the sinc-Lorentzian pulse. This improvement confirms the gain observed in the effective SNR as shown in Fig. (5). When $\delta t$ is reduced to $0.05 T_{s}$, an improvement of about $95 \%$ in the BER can be achieved. Additionally, in Fig. 7(b), at $\delta t=0.35 T_{s}$, the BER performance of the sinc-Lorentzian pulse is further improved by about an order of magnitude compared to the RC and the BTRC pulses. Whilst the RC and the BTRC pulses show a minimum BER of $3.2 \times 10^{-3}$ at $\delta t=0.05 T_{s}$, the sinc-Lorentzian pulse shows error-free transmission, i.e., zero BER, for $0 \leq \delta t<0.225 T_{s}$. The eye diagrams in Figs. 7(a) and 7(b) confirm the transmission performance of the three pulse shapes. From these diagrams, the sinc-Lorentzian pulse has the widest eye opening as well as the minimum sidelobe distortion. The superior BER performance exhibited by the proposed pulse is attributed to its low time-domain sidelobe, which is further suppressed by the EO modulation nonlinearity and, hence, reduces the nonlinear interaction between adjacent symbols as illustrated in Fig. 3 .

It is noteworthy that, in contrast to the simulated BER performance shown in Fig. (4), the experimentally evaluated BER performances for the $\mathrm{RC}$ and the BTRC pulse shapes are very close over the entire range of $\delta t$. This discrepancy between the simulated and measured BER performances can be explained as follows. In electrical channels, the ISI is the result of linear interaction between adjacent symbols, i.e., the superposition of the accumulating ISI contributed by all symbols that precede or follow the desired symbol. In optical channels, however, this interaction is affected by the EO conversion nonlinearity. Additionally, the SBD is one of the highly-nonlinear elements in terahertz wireless links, which further strengthens the nonlinear interactions between adjacent symbols [24]. At the receiver side, matched filtering is applied to $|p(t)|^{2}$ rather than $p(t)$, which does not result in the maximum SNR, even if the symbols are sampled at the optimum sampling instants. However, such nonlinear interaction does not significantly impact the BER of the proposed waveform due to its rapidly decaying profile.

\section{CONCLUSION}

This paper presents a timing-jitter-tolerant pulse shape for photonically-enabled terahertz communications. The bit error rate performance of the proposed sinc-Lorentzian waveform is numerically and experimentally investigated. Numerical and experimental results show that the proposed pulse shape is more robust than the conventional $\mathrm{RC}$ and BTRC waveforms against the time sampling errors caused by the timing-jitter. The results reported in this work serve as a starting point as well as a guideline for further research on waveform designs that are more robust to the time sampling errors in high-speed photonically-enabled terahertz communications. Furthermore, the proposed waveform can be considered as a baseline to which other newly designed waveforms can be compared.

\section{APPENDIX}

\section{DERIVATION OF THE MCRB FOR THE EFFECTIVE SNR}

Practically, the timing-jitter can be statistically modeled as a random process, which is typically Gaussian [2], with a mean value corresponding to the optimum time sampling instant. The error variance performance of any timing-offset estimator is lower-bounded by the MCRB as follows [23]:

$$
\operatorname{Std}\left(\delta t_{k}\right) \geq \sqrt{\frac{\int_{-\infty}^{\infty}|P(f)|^{2} d f}{4 \pi^{2} \mathrm{SNR}_{\mathrm{eff}} \int_{-\infty}^{\infty} f^{2}|P(f)|^{2} d f}},
$$

where Std (.) is the statistical standard deviation operator, $P(f)$ is the Fourier transform of $p(t)$. Since a band-limited Nyquist pulse does not exist in the optical domain [25], it is more reasonable to express (12) in terms of its RMS spectral 

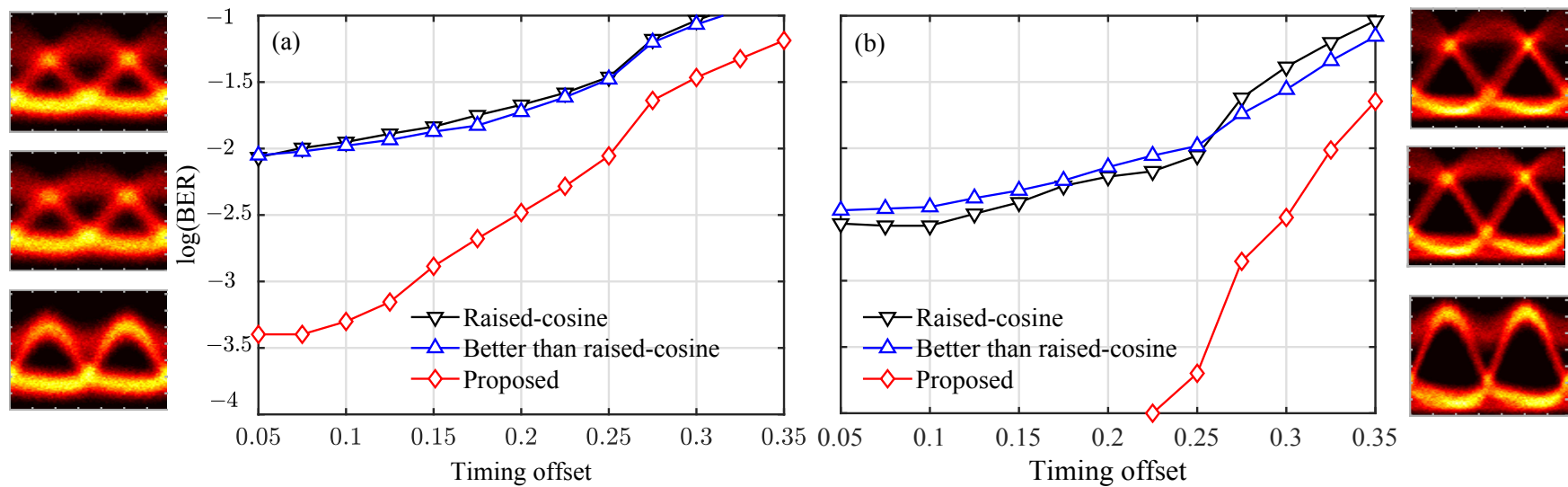

Fig. 7. Bit error rate performance versus the normalized timing-offset of the received waveforms at two different values of the emitted terahertz power. (a) $P_{\mathrm{THz}}=-18.4 \mathrm{dBm}$. (b) $P_{\mathrm{THz}}=-13.3 \mathrm{dBm}$. The eye diagrams are plotted for the RC, BTRC, and the proposed waveforms from top to bottom, respectively, at a normalized timing-offset of 0.05 .

width rather than the Nyquist bandwidth. The RMS spectral width of a given pulse shape, denoted by $\sigma_{f}$, can be defined as follows [26], [27]:

$$
\sigma_{f}=\frac{1}{2 \pi} \sqrt{\left\langle\omega^{2}\right\rangle-\langle\omega\rangle^{2}}
$$

where $\omega=2 \pi f,\langle\omega\rangle$ and $\langle\omega\rangle^{2}$ are first and the second centralized order moments of the pulse spectrum, defined, respectively, as follows:

$$
\begin{aligned}
\left\langle\omega^{2}\right\rangle & =\frac{\int_{-\infty}^{\infty} \omega^{2}|P(\omega)|^{2} d \omega}{\int_{-\infty}^{\infty}|P(\omega)|^{2} d \omega} \\
\langle\omega\rangle & =\frac{\int_{-\infty}^{\infty} \omega|P(\omega)|^{2} d \omega}{\int_{-\infty}^{\infty}|P(\omega)|^{2} d \omega}
\end{aligned}
$$

Since $p(t)$ is real-valued for the three considered waveforms, then $\langle\omega\rangle=0$. Expressing $\sqrt{12}$ in terms of $(13)$ yields:

$$
\mathrm{SNR}_{\mathrm{eff}} \geq \frac{1}{4 \pi^{2} \sigma_{f} \mathbf{S t d}\left(\delta t_{k}\right)}
$$

Assuming that $\mathbf{S t d}\left(\delta t_{k}\right)=\sigma_{t}$ simplifies 15 to the following form:

$$
\mathrm{SNR}_{\mathrm{eff}} \geq \frac{1}{4 \pi^{2} \sigma_{f} \sigma_{t}}
$$

where $\sigma_{t}$ is defined as follows:

$$
\sigma_{t}=\frac{1}{2 \pi} \sqrt{\left\langle t^{2}\right\rangle-\langle t\rangle^{2}}
$$

and

$$
\begin{aligned}
\left\langle t^{2}\right\rangle= & \frac{\int_{-\infty}^{\infty} t^{2}|p(t)|^{2} d t}{\int_{-\infty}^{\infty}|p(t)|^{2} d t}, \\
\langle t\rangle= & \frac{\int_{-\infty}^{\infty} t|p(t)|^{2} d t}{\int_{-\infty}^{\infty}|p(t)|^{2} d t} .
\end{aligned}
$$

\section{REFERENCES}

[1] V. Petrov, T. Kurner, and I. Hosako, "IEEE802.15.3d: first standardization efforts for sub-terahertz band communications toward 6G," IEEE Communications Magazine, vol. 58, no. 11, pp. 28-33, 2020.

[2] G. D. Roumelas, H. E. Nistazakis, E. Leitgeb, A. N. Stassinakis, and G. S. Tombras, "On the performance of optical wireless communication links impaired by time jitter, M-turbulence and pointing errors," Optics Communications, vol. 472, pp. 1-8, 2020.

[3] G. Ducournau, P. Szriftgiser, A. Beck, D. Bacquet, F. Pavanello, E. Peytavit, M. Zaknoune, T. Akalin, and J. F. Lampin, "Ultrawide-bandwidth single-channel $0.4-\mathrm{THz}$ wireless link combining broadband quasi-optic photomixer and coherent detection," IEEE Transactions on Terahertz Science and Technology, vol. 4, no. 3, pp. 328-337, 2014.

[4] T. Nagatsuma, S. Horiguchi, Y. Minamikata, Y. Yoshimizu, S. Hisatake, S. Kuwano, N. Yoshimoto, J. Terada, and H. Takahashi, "Terahertz wireless communications based on photonics technologies," Optics Express, vol. 21, no. 20, pp. 23736-23 747, 2013.

[5] T. Nagatsuma, Y. Fujita, Y. Yasuda, Y. Kanai, S. Hisatake, M. Fujiwara, and J. Kani, "Real-time 100-Gbit/s QPSK transmission using photonicsbased 300-GHz-band wireless link," International Topical Meeting on Microwave Photonics (MWP), vol. 1, pp. 27-30, 2016.

[6] J. Webber, N. Nishigami, J. Y. Kim, M. Fujita, and T. Nagatsuma, "Terahertz wireless communications using resonant tunnelling diodes with radio-over-fibre," Electronics Letters, vol. 55, no. 17, pp. 949-951, 2019.

[7] V. K. Chinni, P. Latzel, M. Zégaoui, C. Coinon, X. Wallart, E. Peytavit, J. F. Lampin, K. Engenhardt, P. Szriftgiser, M. Zaknoune, and G. Ducournau, "Single-channel $100 \mathrm{Gbit} / \mathrm{s}$ transmission using III-V UTC-PDs for future IEEE 802.15.3d wireless links in the $300 \mathrm{GHz}$ band," Electronics Letters, vol. 54, no. 10, pp. 638-640, 2018.

[8] V. K. Chinni, M. Zezaoui, C. Coinon, X. Wallart, E. Pevtavit, J. F. Larnpin, P. Szriftgiser, M. Zaknoune, and G. Ducournau, "Indoor 100 Gbit/s THz data link in the $300 \mathrm{GHz}$ band using fast photodiodes," 25th International Conference on Telecommunications, ICT, pp. 288290, 2018. 
[9] M. Fujishima, "Key technologies for THz wireless link by silicon CMOS integrated circuits," Photonics, vol. 5, no. 4, pp. 1-17, 2018.

[10] I. Dan, G. Ducournau, S. Hisatake, P. Szriftgiser, R. P. Braun, and I. Kallfass, "A terahertz wireless communication link using a superheterodyne approach," IEEE Transactions on Terahertz Science and Technology, vol. 10 , no. 1 , pp. 32-43, 2020.

[11] N. C. Beaulieu, "The evaluation of error probabilities for intersymbol and cochannel interference," IEEE Transactions on Communications, vol. 39, no. 12, pp. 1740-1749, 1991.

[12] "IEEE Standard for High Data Rate Wireless Multi-Media Networks-Amendment 2: $100 \mathrm{~Gb} / \mathrm{s}$ Wireless Switched Point-to-Point Physical Layer," IEEE Std 802.15.3d-2017 (Amendment to IEEE Std 802.15.3-2016 as amended by IEEE Std 802.15.3e-2017), pp. 1-55, 2017.

[13] M. Shehata, K. D. Wang, J. Webber, M. Fujita, T. Nagatsuma, and W. Withayachumnanku, "IEEE 802.15.3d-compliant waveforms for terahertz wireless communications," April 2021. [Online]. Available: https://www.techrxiv.org/articles/preprint/IEEE_802_15_3d-Compliant_ Waveforms_for_Terahertz_Wireless_Communications/14393093

[14] N. S. Alagha and P. Kabal, "Generalized raised-cosine filters," IEEE Transactions on Communications, vol. 47, no. 7, pp. 989-997, 1999.

[15] H. Nyquist, "Abridgment of certain topics in telegraph transmission theory," Journal of the American Institute of Electrical Engineers, vol. 47, no. 3, pp. 214-217, 1928.

[16] N. C. Beaulieu, C. C. Tan, and M. O. Damen, "A "better than" Nyquist pulse," IEEE Communications Letters, vol. 5, no. 9, pp. 367-368, 2001.

[17] A. Assalini and A. M. Tonello, "Improved Nyquist pulses," IEEE Communications Letters, vol. 8, no. 2, pp. 87-89, 2004.

[18] A. Kumar, M. Magarini, and S. Bregni, "Improving GFDM symbol error rate performance using better than Nyquist pulse shaping filters," IEEE Latin America Transactions, vol. 15, no. 7, pp. 1244-1249, 2017.

[19] P. A. Haigh, P. Chvojka, S. Zvánovec, Z. Ghassemlooy, and I. Darwazeh, "Analysis of Nyquist pulse shapes for carrierless amplitude and phase modulation in visible light communications," Journal of Lightwave Technology, vol. 36, no. 20, pp. 5023-5029, 2018.

[20] B. Farhang-Boroujeny and G. Mathew, "Nyquist filters with robust performance against timing jitter," in Proceedings of GLOBECOM'96. 1996 IEEE Global Telecommunications Conference, vol. 2, 1996, pp. $1335-1339$.

[21] S. D. Assimonis, M. Matthaiou, and G. K. Karagiannidis, "Twoparameter Nyquist pulses with better performance," IEEE Communications Letters, vol. 12, no. 11, pp. 807-809, 2008.

[22] T. Koike-Akino, D. S. Millar, K. Parsons, and K. Kojima, "Evolutionary design of pulse-shaping FIR filter to mitigate fiber nonlinearity," in Advanced Photonics 2018 (BGPP, IPR, NP, NOMA, Sensors, Networks, SPPCom, SOF), vol. Part F112-SPPCom 2018, 2018, pp. 1-2.

[23] A. N. D'Andrea, U. Mengali, and R. Reggiannini, "The modified Cramer-Rao bound and its application to synchronization problems," IEEE Transactions on Communications, vol. 42, no. 234, pp. 13911399, 1994

[24] T. Harter, C. Füllner, J. N. Kemal, S. Ummethala, J. L. Steinmann, M. Brosi, J. L. Hesler, E. Bründermann, A. S. Müller, W. Freude, S. Randel, and C. Koos, "Generalized Kramers-Kronig receiver for coherent terahertz communications," Nature Photonics, vol. 14, no. 10, pp. 601-606, 2020.

[25] S. Hranilovic, "Minimum-bandwidth optical intensity Nyquist pulses," IEEE Transactions on Communications, vol. 55, no. 3, pp. 574-583, 2007.

[26] L. Cohen, "The uncertainty principle in signal analysis," in Proceedings of IEEE-SP International Symposium on Time- Frequency and TimeScale Analysis, 1994, pp. 182-185.

[27] P. J. Loughlin and L. Cohen, "The uncertainty principle: Global, local, or both?" IEEE Transactions on Signal Processing, vol. 52, no. 5, pp. 1218-1227, 2004. 